\title{
OPTIMIZATION AND INVESTIGATION OF MULTI-STAGE THERMOELECTRIC GENERATION SYSTEMS WITH PARTICLE SWARM OPTIMIZATION (PSO)
}

\author{
Alemi.H , Aghanajafi.C and Kashi. $A^{1}$ \\ K.N. Toosi university of Technology, Tehran, Iran
}

\begin{abstract}
The aim of this research is to investigate the optimization of some properties of thermoelectric systems in generators systems. The procedures used for the optimization of the system are the particle swarm optimization (PSO). First, the maximum efficiency and power production in multi-stage thermoelectric systems, which are connected in both parallel and series, are computed. Then, the performance of these systems is compared with each other. After that the priorities of their usage at their operating temperatures are determined. In this study, despite other studies in which thermoelectric characteristics of materials are calculated at the average temperatures of the system, the characteristic average operating temperatures of each stage is used. The procedure used for optimizing the system is the particle swarm optimization method (PSO) which is a really powerful and fast solution especially in continuous problems.
\end{abstract}

Key words: Optimization theory, Thermoelectric systems (electric field), Multi-stage systems, Electric Efficiency, Electrical Generated Power.

\section{Nomenclature}

$T_{h}:$ Temperature of hot surface

$T_{c}$ : Temperature of cold surface

$T_{m 1}$ : Temperature of surface between stage 1 and stage 2 at multi-stage systems

$T_{m 2}$ : Temperature of surface between stage 2 and stage 3 at multi-stage systems

$T_{m 3}:$ Temperature of surface between stage 3 and stage 4 at multi-stage systems

$S$ : seebeck coefficient of a thermoelectric circle

$R$ : electric resistance of a thermoelectric circle

$K$ : heat conductivity of a thermoelectric circle

$G$ : slenderness ratio

I : electric current at series systems

$I_{*} \quad: \quad$ electric current at parallel system at stage(1-4)

$N_{*}:$ number of thermoelectric circle at stage (1-4)

$Q_{\text {in }}:$ inlet heat to system

$Q_{\text {out }}$ : outlet heat from system

$P \quad$ : generation power by system

$\eta$ : efficiency of thermoelectric system

DOI : 10.14810/elelij.2014.3201 
Electrical and Electronics Engineering: An International Journal (ELELIJ) Vol 3, No 2, May 2014

\section{Introduction}

According to recent estimations, fossil fuels will run out in sixty four years, therefore, people will have to turn to new sources of energy.

Using renewable energy such as hydro, wind and solar are among the most accepted, and improving their efficiency and performance has attracted scientists' interest [1]. One method is using energy producing devices with solid state [2]. These devices have low maintenance cost and they operate without polluting the environment.

Thermoelectric devices are divided into two major groups: coolers [3-6] and generators [7-10]. Coolers operate on the basis of Peltier effect and generators operate on the basis of Seebeck effect. Thermoelectric generators produce electricity when there is a temperature difference, and thermoelectric coolers produce a temperature difference in the presence of electricity. In the present time, the only limitation of using these devices is their low efficiencies; generators at 5$6 \%$ [11], and the coefficient of performance of coolers is around 1, closer to 4 for a typical system [12].

Thermoelectric materials' characteristics such as thermal conductivity, electrical resistance, seebeck and Peltier coefficients are all functions of temperature [13], which themselves can be topics for researches to investigate.

Yilbas and Sahin suggested a slenderness ratio for maximizing performance [14]. Beside of researches trying to improve thermodynamic material characteristics, a lot of research has done on thermodynamic systems configuration to improve the overall system performance[15-17].

Even with limited performance, some of research effort has led to using these systems in combination of other systems. For example in automotive industry a lot of works have been done in transforming exhaust heat energy to electricity [18-23]. Recently Lee and his colleagues have developed a solar-thermoelectric system which has attracted much attention [24]. They have found $\mathrm{Bi} 2 \mathrm{Te} 3$, considering its operating temperature, is the best material for a solarthermoelectric system . In solar energy, Wei He analyzed a design of a heat pipe solar-electric generator unit[25].

Particle swarm optimization is a new procedure in solving optimization problems [26]. In this algorithm, there are some things which are called particles and are distributed in the searching space of the function which has to be optimized Each particle calculates the amount of the cost function in its own location within the space. Then, by combining two sets of information of the present location and the best previous location where it used to be, and also, the information of one or some of the best particles of the group, it chooses a direction to move. This method uses low order relation, which helps the result to converge faster. In this paper, we used violation technique to satisfy the boundary conditions of the problem. In the PSO method, the motions of the particles are a direct result of adding three components such as global -search, local search, and the previous traveled distance which is multiplied by by a set of certain weighting coefficients. In this case, the method to search for the particle could be modified by manipulating the weighting coefficients, but it is better to conduct a wide global search first, and then a local search around the most likely location.

In PSO method, finding optimal weights for faster convergence, accurate space search and better performance is a challenging problem for scientists [27]. 
This research looks for an optimal arrangement of Single-stage through four-stage thermo-electric generators, taking their operating temperatures into consideration. Different stages of the system are connected to each other in parallel and in series, and the results of these systems are compared to each other. $\mathrm{Bi}_{2} \mathrm{Te}_{3}$ is used as the thermoelectric material which is a very useful and practical substance for this application.

\section{Thermoelectric materials' work procedure}

When an electrical conductor is positioned between two different temperatures, the conductor has the ability to transfer the thermal energy from the hot spot to the cold one. In addition, the physical procedure of heat transfer tends to transfer the electrical charge carriers in the direction of the heat transfer.

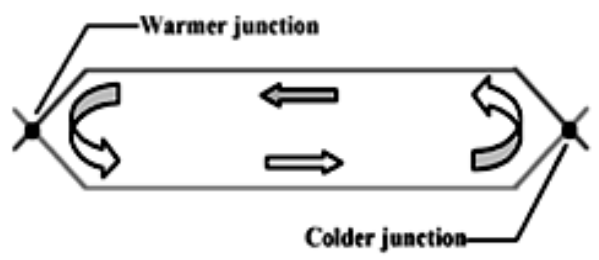

Figure 1. Schema of a simple circuit with 2 wire

This movement of charge carriers can be used to produce electrical current, if we are able to complete the circuit in an effective way. If the conductor completing the circuit is like the first conductor, the flow of the thermal energy produces an electrical potential for charge carriers' movements in both conductors. Moreover, the electrical potential in one of the conductor is exactly the opposite of that of the other conductor.

As a result, there will be no current in the circuit. If we use two different conductors, we will have a completely different result. With different abilities of transferring carriers, the produced current resulted from thermal potential in one conductor will dominate the produced current resulted from thermal potential of the other conductor (and in some cases it will completely dominate the other current).

The pure effect is a continuous current which is the difference between the produced current of the two conductors. The presence of this pure current shows that there is an electrical potential along the path of the heat flow which could be easily measure by opening the circuit and using a potentiometer. Notice that the ability of different materials in producing voltage by presence of a temperature difference is called seebeck effect. The produced voltage, also, is called seebeck voltage. In fact, the produced voltage by the thermocouple is a function of two parameters: the temperature difference between the two points and the characteristics of the used conductors (like dependence on temperature). Of course, thermocouples are used to measure temperatures not to produce power. 


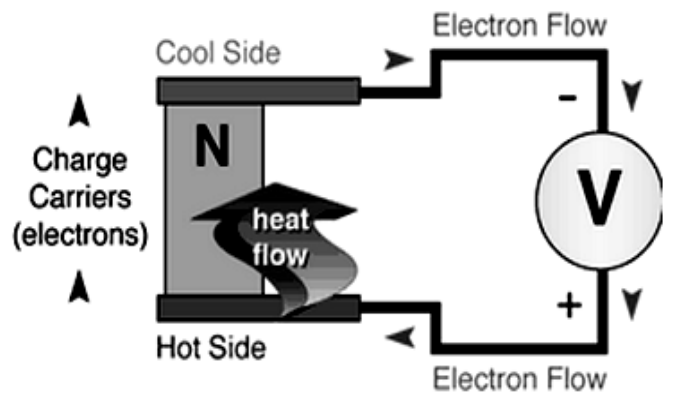

Figure 2. Schema of N-type circle include the voltmeter

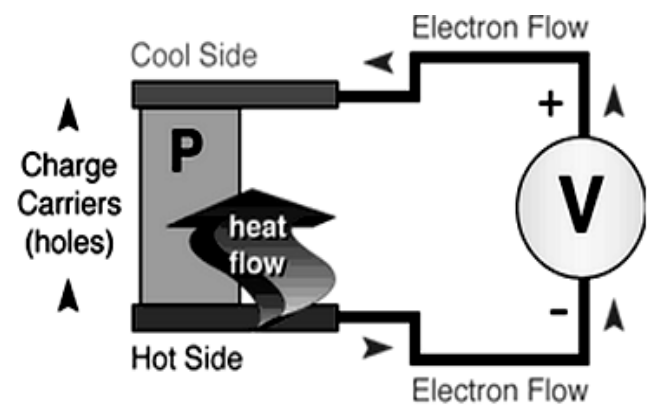

Figure 3 Schema of P-type circle include the voltmeter

Thermoelectric power producing facilities use semi-conductor materials for which the seebeck effect is optimized.

The circuit in fig.(2) shows a simple sample. An N-type semi-conductor circle connected to a voltmeter is shown in this figure. When heat is transferred from the hot spot to the cold spot, charge carriers are transferred with heat. Heat, also, causes the movement of charge carriers in the return path. For producing power in thermo-electrics, $\mathrm{P}$ circle are used too.

Figure (3) shows a primary schematic. Notice the reverse movement of electrons. These are all of the cases of using type- $\mathrm{N}$ and $\mathrm{P}$ materials in a power producing generator which we can correctly optimize the seebeck effect.

As it is shown in fig.(4), $\mathrm{N}$ and $\mathrm{P}$ circle are in a parallel position, but are circle from an electrical point of view. Because the electrical current (electrons' movements) is in the opposite direction of the cavity current, the current producing potentials in circle are not each others' opposite

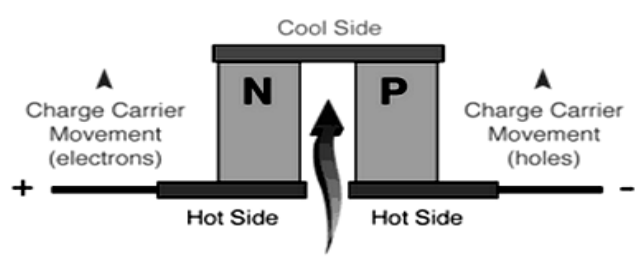

Figure 4. Schema of parallel type

In fact, in practical PEGs, a lot of $\mathrm{N}$ and $\mathrm{P}$ couples are used to gain applicable voltage 


\section{Defining the model}

\subsection{Schematic and structural definitions of the model}

Structural model of thermoelectric systems is presented in Fig.(5):

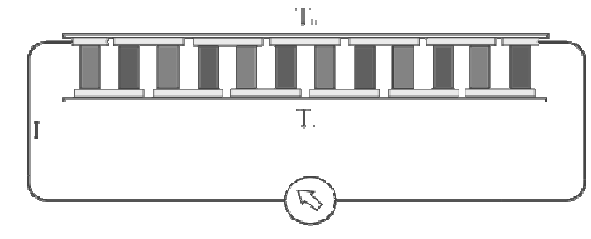

(a)

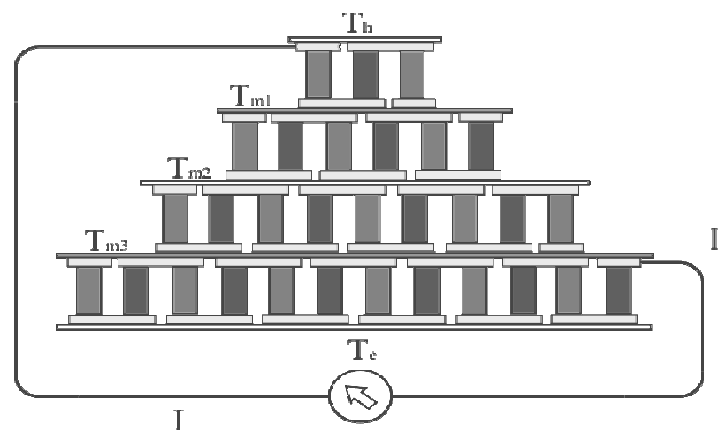

(d)

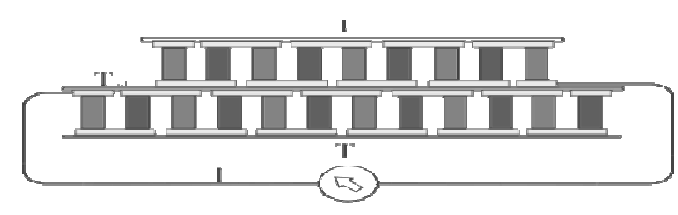

(b)

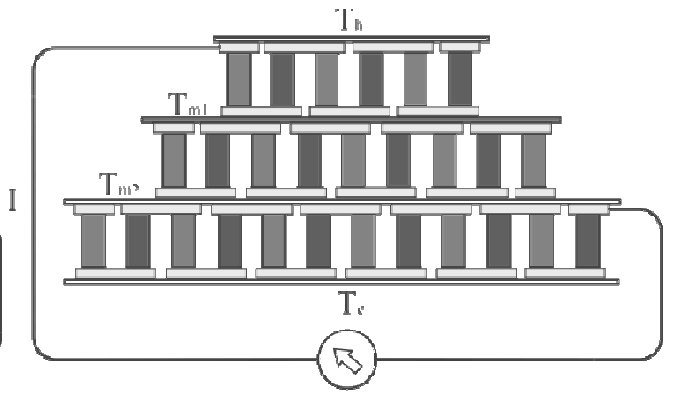

Fig.(5): the thermoelectric system,(a) the single-stage system,(b) the two-stage series system,(c) the threestage series system,(d) the four-stage series system

The two-stage series system is composed of two single-stage systems which are on top of one another, connected by a wire in series. This concept of two-stage thermoelectric systems can be extended to three- and four-stage thermoelectric systems, as shown in Fig. 5 (c) and (d).

Structural model of parallel thermoelectric systems is shown in Fig.(6).

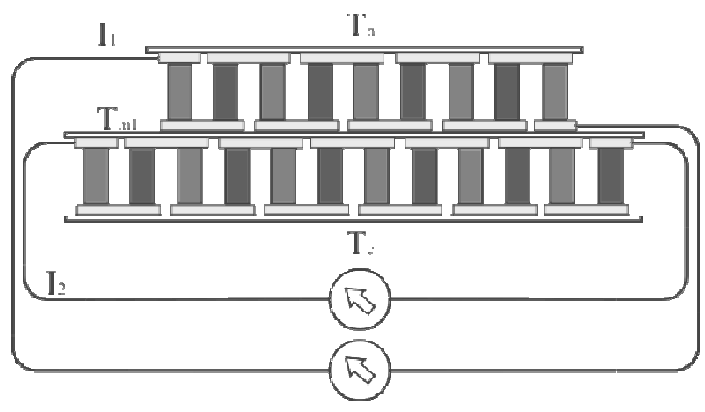

(a)

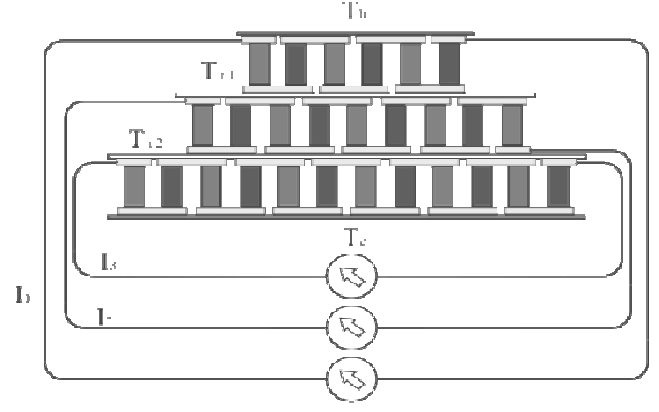

(b) 


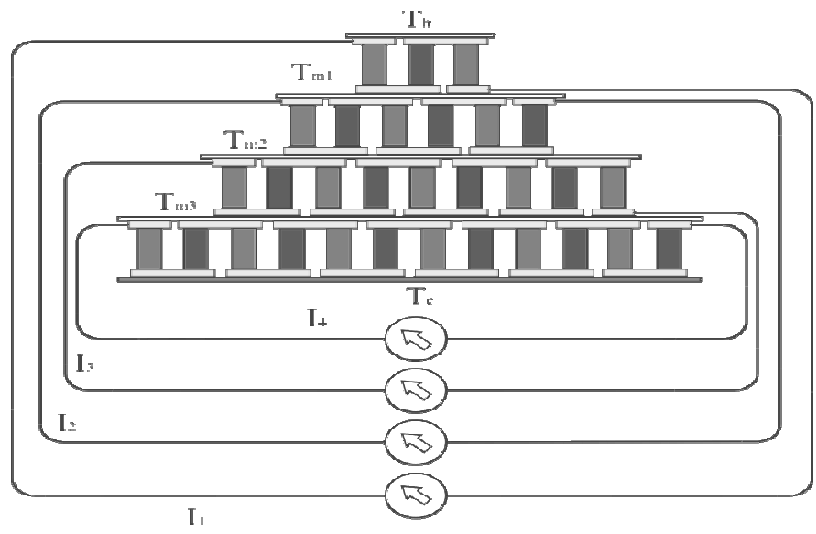

(c)

Fig.(6): the parallel thermoelectric system, (a) the two-stage system, (b) the three-stage system, and (c) the four-stage system

Multi-stage parallel thermoelectric systems are similar to the series thermoelectric systems with only one difference, which is, the produced power enters the circuit in each stage.

\subsection{Mathematical definition of the model}

The system's inlet heat, outlet heat, and work in a single-stage thermoelectric system are calculated using the second law of thermodynamics as follows [28]:

$Q_{\text {in }}=N \times\left(S T_{h} I-\frac{1}{2} R_{\text {in }} I^{2}+K\left(T_{h}-T_{c}\right)\right)$
$Q_{\text {out }}=N \times\left(S T_{c} I+\frac{1}{2} R_{\text {in }} I^{2}+K\left(T_{h}-T_{c}\right)\right)$

The cost functions in this study are the system's efficiency and produced power, which are based on thermodynamic laws. The produced power of the system are calculated as follows:

$P=Q_{\text {in }}-Q_{\text {out }}=N \times\left(S I\left(T_{h}-T_{c}\right)-R_{\text {in }} I^{2}\right)$

Also, the systems efficiency is given by:

$\eta=\frac{P}{Q_{\text {in }}}=\frac{N \times\left(S I\left(T_{h}-T_{C}\right)-R_{\text {in }} I^{2}\right)}{N \times\left(S T_{h} I-\frac{1}{2} R_{\text {in }} I^{2}+K\left(T_{h}-T_{c}\right)\right)}$

In order to extend the above equations to multi-stage systems, these equations must be applied to each stage. Considering the thermodynamic condition emphasizing the equivalence of the inlet and outlet heat transfer during each stage, at the end, equations (3) and (4) are applied to the whole system. In this case, the lender coefficient of the thermoelectric couple, are considered to be: $G=5 \times 10^{-3}(\mathrm{~m})$.

In a parallel two-stage thermoelectric generator, there are five optimization variables as follow: $\mathrm{T}_{\mathrm{m}}, \mathrm{I}_{1}, \mathrm{I}_{2}, \mathrm{~N}_{1}, \mathrm{~N}_{2}$ In a series two-stage thermoelectric generator, I1 equals I2; as a result, the number of optimization variables reduces to four. Thermodynamic condition in two-stage systems 
is $Q_{\text {outlet from stage } 1}=Q_{\text {inlet to stage } 2}$ which should be satisfied during the optimization process.

In a parallel three-stage thermoelectric system, there are eight optimization variables as follow: $\mathrm{T}_{\mathrm{m} 1}, \mathrm{~T}_{\mathrm{m} 2}, \mathrm{I}_{1}, \mathrm{I}_{2}, \mathrm{I}_{3}, \mathrm{~N}_{1}, \mathrm{~N}_{2}, \mathrm{~N}_{3}$ In a series three-stage thermoelectric system $\mathrm{I}_{1}, \mathrm{I}_{2}$, and $\mathrm{I}_{3}$ are equal; as a result, the number of optimization variables reduces to six. Thermodynamic conditions in three-stage systems are $Q_{\text {outlet from stage } 1}=Q_{\text {inlet to stage } 2}$ and $Q_{\text {oulet from stage } 2}=$ $Q_{\text {inlet to stage3 }}$ which should be satisfied during the optimization process.

In a parallel four-stage thermoelectric system, there are eleven optimization variables as follow: $\mathrm{T}_{\mathrm{m} 1}, \mathrm{~T}_{\mathrm{m} 2}, \mathrm{~T}_{\mathrm{m} 3}, \mathrm{I}_{1}, \mathrm{I}_{2}, \mathrm{I}_{3}, \mathrm{~N}_{1}, \mathrm{~N}_{2}, \mathrm{~N}_{3}$. In a series four-stage thermoelectric system $\mathrm{I}_{1}, \mathrm{I}_{2}, \mathrm{I}_{3}$, and $\mathrm{I}_{4}$ are equal; as a result, the number of optimization variables reduces to eight. Thermodynamic conditions in four-stage systems are $Q_{\text {outlet from stage } 1}=Q_{\text {inlet to satage } 2}$ and $Q_{\text {outlet from stage } 2}=Q_{\text {inlet to sateg } 3}$ and $Q_{\text {outlet from stage } 3}=Q_{\text {inlet to stage } 4}$ which should be satisfied during the optimization process.

For example, the equations of entering and exiting heats in a parallel three-stage thermo-electric system are:

$$
\begin{aligned}
& Q_{1, \text { in }}=N_{1} \times\left(S_{1} T_{h} I_{1}-\frac{1}{2} R_{1} I_{1}{ }^{2}+K_{1}\left(T_{h}-T_{m 1}\right)\right) \\
& Q_{1, \text { out }}=N_{1} \times\left(S_{1} T_{m 1} I_{1}+\frac{1}{2} R_{1} I_{1}{ }^{2}+K_{1}\left(T_{h}-T_{m 1}\right)\right) \\
& Q_{2, \text { in }}=N_{2} \times\left(S_{2} T_{m 1} I_{2}-\frac{1}{2} R_{2} I_{2}{ }^{2}+K_{2}\left(T_{m 1}-T_{m 2}\right)\right) \\
& Q_{2, \text { out }}=N_{2} \times\left(S_{2} T_{m 2} I_{2}+\frac{1}{2} R_{2} I_{2}{ }^{2}+K_{2}\left(T_{m 1}-T_{m 2}\right)\right) \\
& Q_{3, \text { in }}=N_{3} \times\left(S_{3} T_{m 2} I_{3}-\frac{1}{2} R_{3} I_{3}{ }^{2}+K_{3}\left(T_{m 2}-T_{c}\right)\right) \\
& Q_{3, \text { out }}=N_{3} \times\left(S_{3} T_{c} I_{3}+\frac{1}{2} R_{3} I_{3}{ }^{2}+K_{3}\left(T_{m 2}-T_{c}\right)\right) \\
& P=Q_{1, \text { in }}-Q_{3, \text { out }} \\
& \eta=\frac{P}{Q_{1 \text { sin }}}
\end{aligned}
$$

If we substitute I for $\mathrm{I}_{1}, \mathrm{I}_{2}$, and $\mathrm{I}_{3}$ in equations (5) to (10), the equations of a series thermoelectric system could be obtained.

Characteristics of the thermoelectric material $\mathrm{Bi}_{2} \mathrm{Te}_{3}(\mathrm{~K}, \mathrm{R}, \mathrm{S})$ are available in [29].

In this case, the following equation is used for the number of thermoelectric couples:

$\sum_{i=1}^{j=N u m b e r}$ of stages $\quad N_{i}=100$

\section{Particle swarm optimization}

Particle swarm optimization is a new procedure in solving optimization problems [26]. In this algorithm, there are some things which are called particles and are distributed in the searching space of the function which has to be optimized each particle calculates the amount of the cost function in its own location within the space. Then, by combining two sets of information of the present location and the best previous location where it used to be, and also, the information of one or some of the best particles of the group, it chooses a direction to move. This method uses 
low order relation, which helps the result to converge faster. In this paper, we used violation technique to satisfy the boundary conditions of the problem.

In the PSO method, the motions of the particles are a direct result from adding three components such as global -search, local search, and the previous traveled distance which is multiplied by a set of certain weighting coefficients. In this case, the method to search for the particle could be modified by manipulating the weighting coefficients, but it is better to conduct a wide global search first, and then a local search around the most likely location. In PSO method, finding optimal weights for faster convergence, accurate space search and better performance is a challenging problem for scientists [27].

This research looks for an optimal arrangement of Single-stage through four-stage thermoelectric generators, taking their operating temperatures into consideration. Different stages of the system are connected to each other in parallel and in series, and the results of these systems are then compared to each other. $\mathrm{Bi}_{2} \mathrm{Te}_{3}$ is used as the thermoelectric material which is a very useful and practical substance for this application.

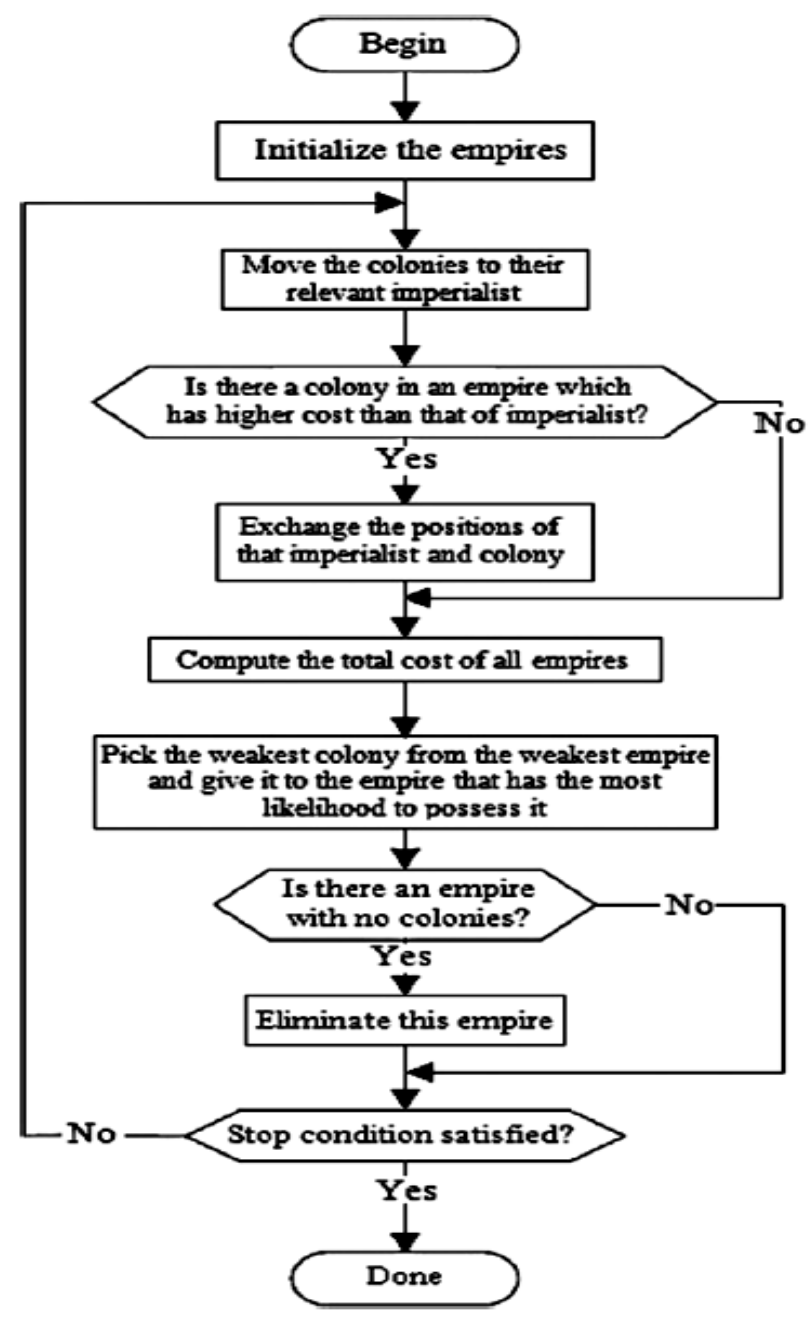

Figure 9. Flowchart of PSO algorithm. 


\section{Results and Discussion}

The obtained mathematical equations presented in mathematical model section were optimized under the specified conditions, and the values of the desired cost function were calculated, and the independent parameters associated with the values were found. Now, the results of series and parallel thermoelectric systems are going to be compared.

\subsection{Impact of the variables on the system's efficiency}

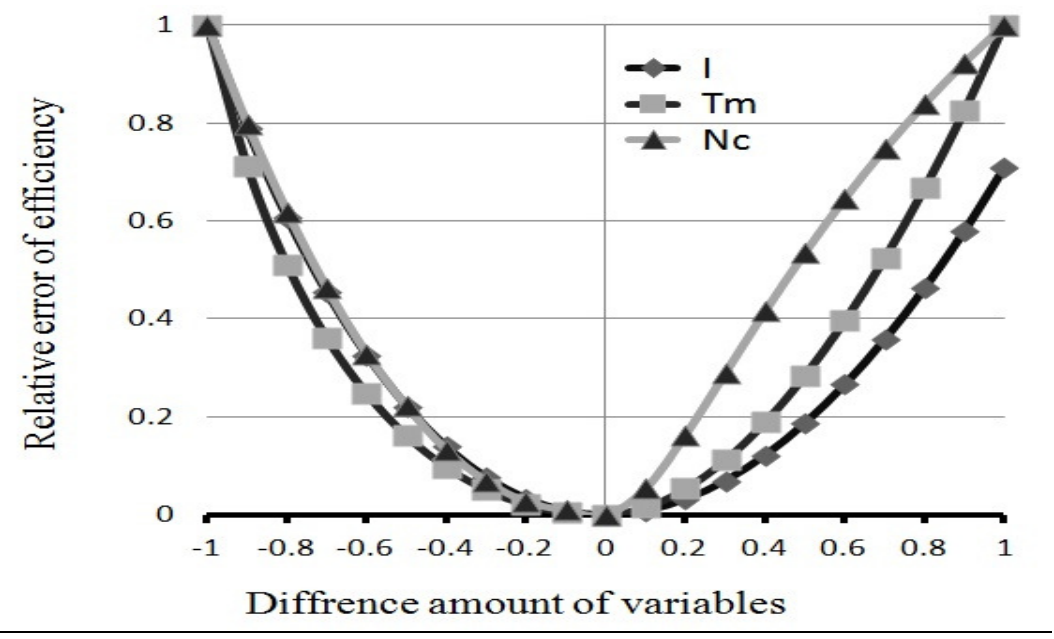

Fig.(7): the effect of optimization variables on the optimized efficiency of the system

As it is shown in figure 7, the system's sensitivity to $\mathrm{N}_{\mathrm{c}}$ and $\mathrm{N}_{\mathrm{h}}$ is higher than its sensitivity to other variables, and this fact is even clearer when $\mathrm{N}_{\mathrm{c}}$ is higher than its optimized value. Figure 7 also indicates that when $\mathrm{T}_{\mathrm{m}}$ and $\mathrm{I}$ have lower values than their optimized ones, I has a larger effect on the efficiency, but when they are increased to levels higher than their optimized values, $\mathrm{T}_{\mathrm{m}}$ has a larger effect on the efficiency.

\subsection{Reasons of efficiency and power decrease by increasing temperature}

As it was observed from the obtained results, the efficiency (at the temperatures higher than $700 \mathrm{~K}$ ) and the produced power (at temperatures higher than $1000 \mathrm{~K}$ ) decline. The reasons for this behavior will be discussed further. 
Electrical and Electronics Engineering: An International Journal (ELELIJ) Vol 3, No 2, May 2014

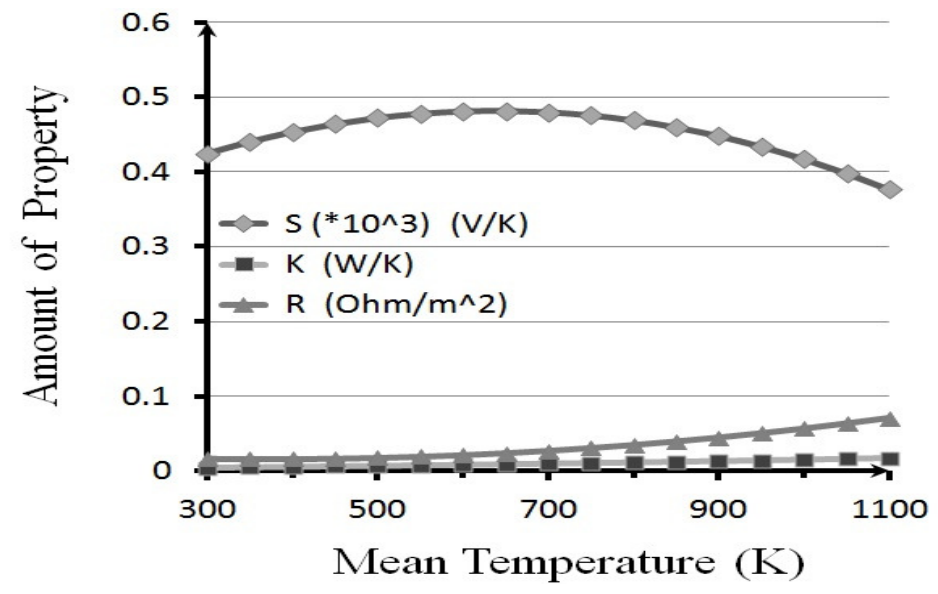

Fig.(8): Changes of the characteristics of the thermoelectric material $\left(\mathrm{Bi}_{2} \mathrm{Te}_{3}\right)$ with mean temperature of hot surface and cold surface

thermoelectric properties obtained by using the average temperatures of hot and cold surfaces. From fig.(8), it can be seen that $\mathrm{R}$ and $\mathrm{K}$ variables ascend as the temperature increases, but the $\mathrm{S}$ variable (seebeck coefficient) starts to decrease once the mean temperature exceeds $650 \mathrm{~K}$. In solving the governing equations of the inlet and outlet heat and the produced power in a singlestage thermoelectric system (eq (1),(2) and (3)), the $\mathrm{K}$ variable is eliminated in the equation, which calculates the produced power.

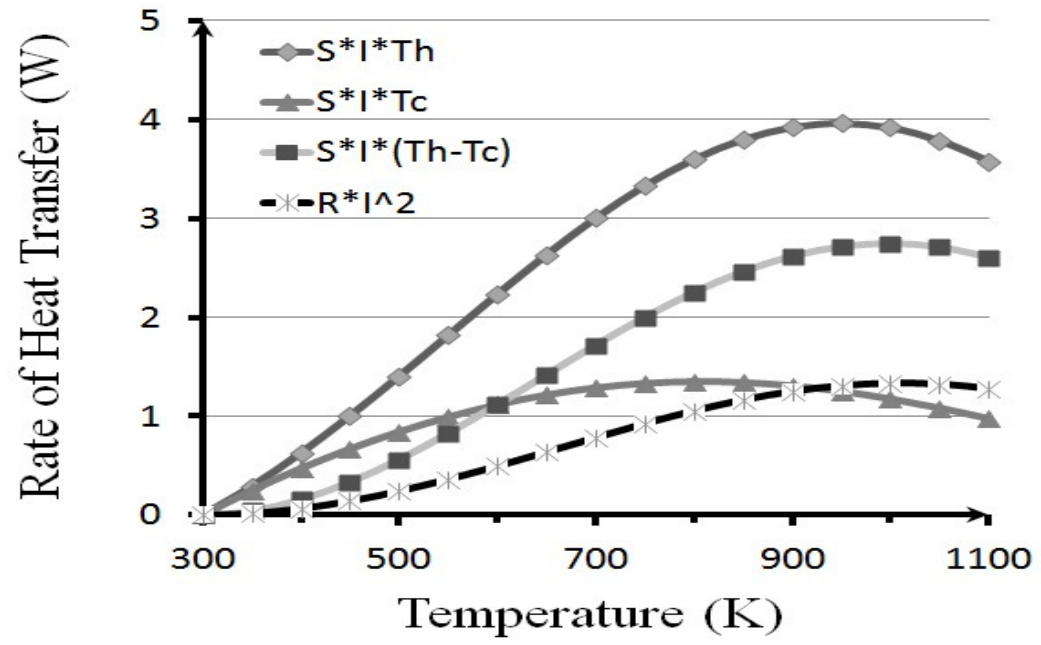

Fig.(9): effect of differences of constituted elements of system on rate of heat transfer

The reason why the produced power decreases at temperatures higher than $1000 \mathrm{~K}$ is because the seebeck coefficient decreases, resulting in the decrease of the term $\operatorname{SI}\left(\mathrm{T}_{\mathrm{h}}-\mathrm{T}_{\mathrm{c}}\right)$. The ascending behavior of $\mathrm{R}$ and the descending behavior of $\mathrm{S}$ at mean temperatures above $650 \mathrm{~K}$, also have an impact on the rate at which power is produced.

As it was mentioned earlier, the efficiency of a thermoelectric system equals eq(4)

The numerator of this equation behaves exactly the same as the procedure mentioned in the previous section, but in the denominator, the rate of conductive heat transfer is added to the other effective elements of the system's power. As it can be seen in figure 7, the K coefficient has 
ascending behavior with respect to temperature. In addition, the term $\left(T_{h}-T_{c}\right)$ has ascending behavior as the surface temperature increases; as a result, the amount of the conductive heat transfer dramatically increases which makes the denominator grow faster than the numerator which leads to the decreasing behavior of the efficiency at temperatures above $700 \mathrm{~K}$.

The same behavior can be observed in multi-stage thermoelectric systems. However, in multistage systems the common part temperature of the stages can be determined in order to decrease the undesired effect of decreasing seebeck coefficient on the ascending heat conduction which leads to lower efficiency. Not to mention that the descending slope of the efficiency could be decreased as well.

It is also worth mentioning that in order to improve the thermoelectric characteristics of materials, the produced power, the efficiency of the system and the seebeck coefficient should be increased, and the electrical resistance coefficient along with the thermal conductivity should be decreased.

\subsection{Optimizing the efficiency of the system}

As it can be seen in figure 10, in series thermo-electric systems, the efficiency of the system has approximately the same value in single-, two-, three-, and four-stage thermo-electric systems until the hot surface temperature reach $600 \mathrm{~K}$ (assuming the cold surface has a temperature of $300 \mathrm{~K}$ ). However, as the temperature of the hot surface increases beyond $600 \mathrm{~K}$, the efficiency of the single-stage system decreases dramatically, and around $1600 \mathrm{~K}$, the efficiency and produced power approach zero. On the other hand, in two-, three-, and four-stage thermo-electric systems, although the efficiency decreases at higher temperatures, its slope is far less than that of the single-stage system.

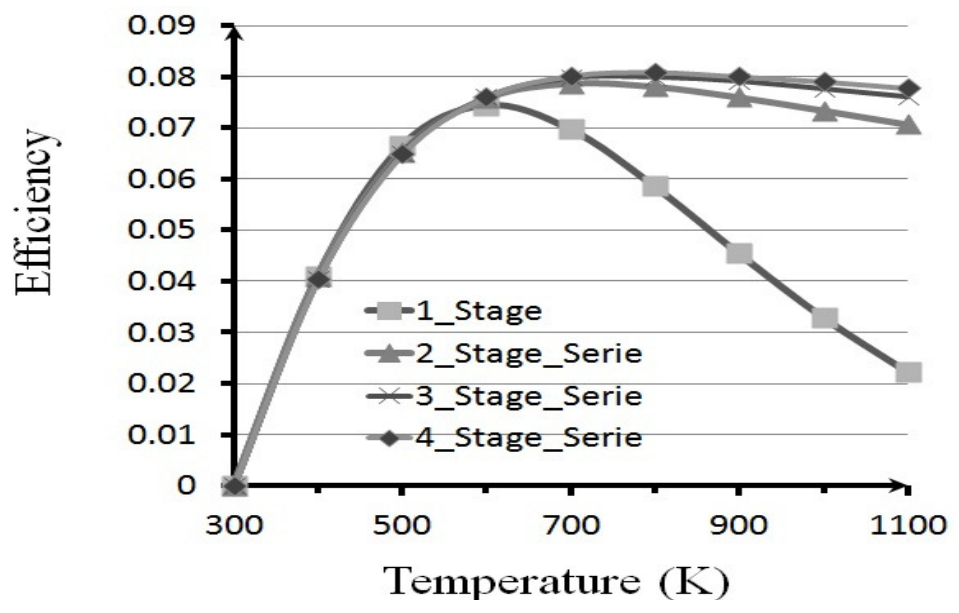

Fig.(10): the effect of hot surface's temperature and number of stages in a series thermo-electric system 


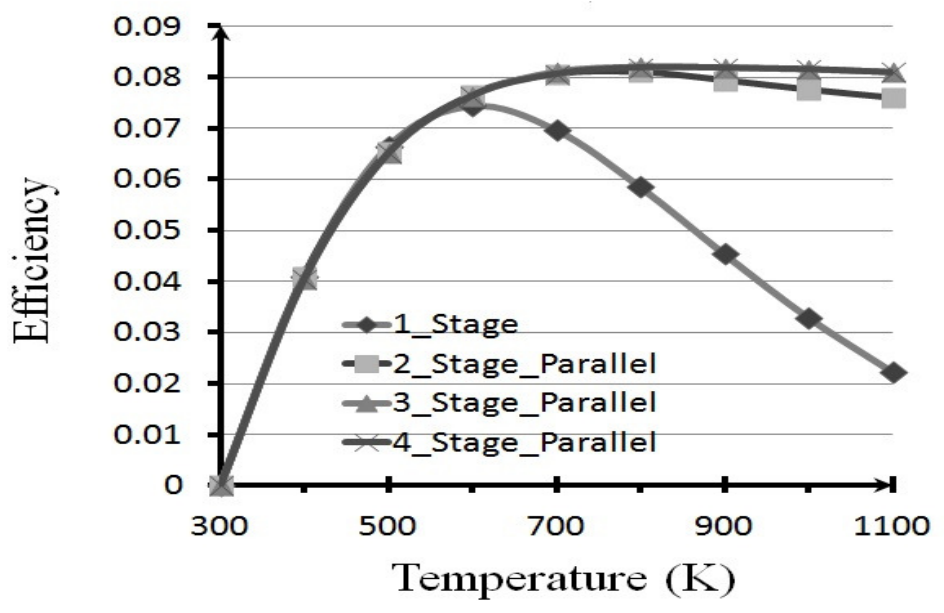

Fig.(11): the effect of hot surface's temperature and number of stages in a parallel thermo-electric system

By comparing figures (10) and (11), it could be determined that the behavior of the parallel thermo-electric system is highly similar to that of the series system. Like series thermo-electric systems, in parallel thermo-electric systems, the maximum efficiency is independent of the number of stages till it reaches the temperature of $600 \mathrm{~K}$, but at temperatures higher than $600 \mathrm{~K}$, the maximum efficiency in the single-stage thermo-electric decreases dramatically and loses its advantages over the systems with more than one stage. It is clear that in multi-stage parallel thermo-electric systems, the efficiency is slightly higher than that of the series systems. By increasing the number of stages, not only the efficiency increase, but also less power is obtained for 100 thermo-electric couples, which is an important advantage of these systems, where we have constraints on space.

\subsection{Optimizing the generated power of the system}

As it is shown in figure 12, the maximum power among the series thermo-electric systems is produced in the single-stage thermo-electric system. Where, as the temperature of the hot surface increases up to $1000 \mathrm{~K}$, the produced power has an ascending manner. Beyond $1000 \mathrm{~K}$ the trend begins to decent. As the number of the stages increases to 2 and higher, the overall amount of produced power decrease, but there is no descending trend until the temperature reaches $1100 \mathrm{~K}$.

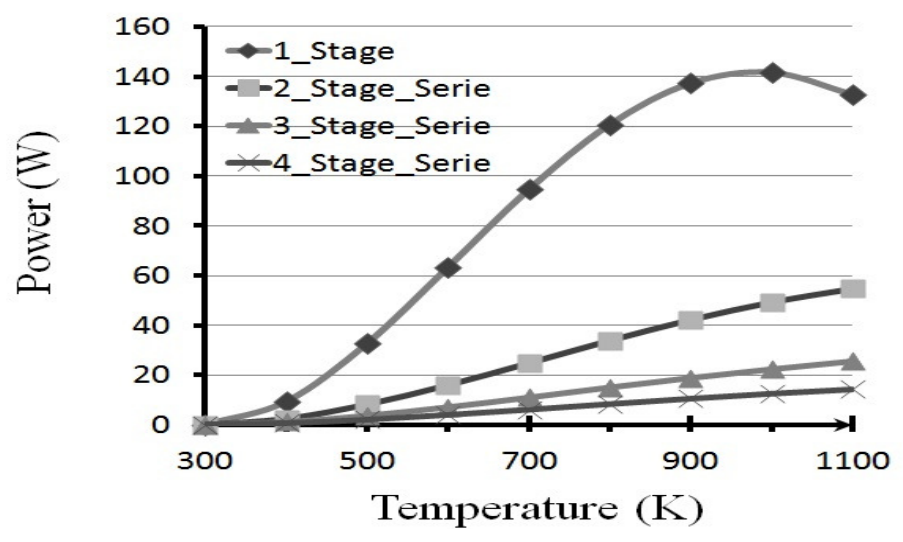

Fig.(12): the effect of the hot surface's temperature and the number of stages on the produced power in Watt in series design 
During efficiency optimization, the parallel thermo-electric system had a better efficiency respect to the series one. The same result was obtained during produced power optimization. The parallel systems with more stages have a higher produced power slope, than that of the series ones. Therefore, the power produced in a parallel system is significantly higher than that of a series system.

It is worth mentioning that the results are obtained when there are 100 couples of thermo-electric series which are divided between the stages when there are several stages in the system. This distribution of thermo-electric couples among different stages results smaller systems, where this is considered as one of important advantages of these systems.

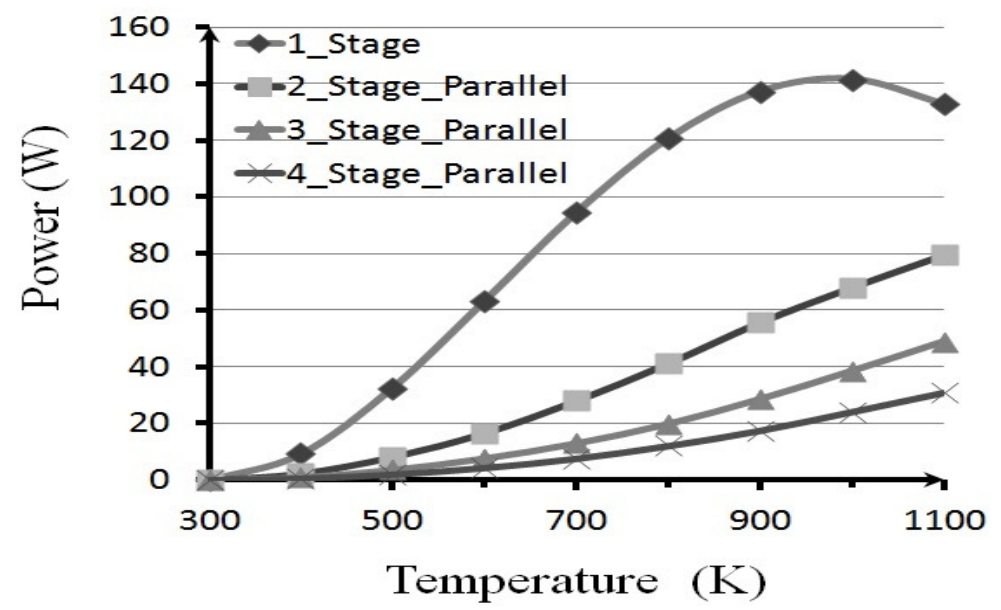

Fig.(13): the effect of the hot surface's temperature and the number of stages on the produced power in Watt in parallel design

\section{Conclusion}

The objective of this study is to find a model of physical and electrical design for thermo-electric systems in order to have the highest efficiency and produced power. Based on the results, the following conclusions are made.

In single-stage systems the hot and cold surface temperatures highly affect the output values of the system; as an example, the efficiency and produced power increase up to $600 \mathrm{~K}$ and $1000 \mathrm{~K}$, respectively.

If the temperature of the hot surface is less than $600 \mathrm{~K}$, using single-stage systems is prefered because there is no noticeable difference between the efficiency of a single-stage system and that of a multi-stage system. However, the amount of power produced is higher for a single-stage system. On the other hand, if the temperature exceeds $600 \mathrm{~K}$, multi-stage systems have a better efficiency. However, the produced power of a single-stage system will still be higher.

Nevertheless, in multi-stage systems with equal number of couples, sometimes the space occupied is half and in some cases one third of the single-stage system which is important when there is a limitation on the available space. 
As it is clear from the results, parallel systems have higher efficiency and produced more power than series systems, and at temperatures higher than $600 \mathrm{~K}$, the associate graph has a lower slope in efficiency decrease, so parallel systems have a more stable performance at higher or variable temperatures although this stability does not guarantee the stable output numbers.

The fact that design and control of multi-stage systems, especially parallel ones, is highly complicated and expensive, should be considered when choosing a system. It could be concluded that even under the best conditions, the output efficiency of a parallel multi-stage system is slightly higher than 8 percent which is not a substantial improvement. So using multi-stage thermo-electric systems over single-stage ones is not advantageous, unless there are some limitations on space, or the purpose is to produce little amount of electricity and the typical systems are not affordable. If the usage of a thermo-electric system is desired, the multi-stage parallel systems is the better choice, given the results of this study. However, this systems are far from ideal. Thanks to the improvements in the realm of nanotechnology, there is hope to improve the thermo-electric characteristics of materials such as the Seebeck effect, the Peltier effect, heat conductivity, and electric resistance; as a result, it is possible in the near future that power production systems, solid state cooling and heating systems such as thermo-electric systems will leave the conventional systems behind in both efficiency and power production.

\section{References}

[1] Rahman S. Going green; "the growth of renewable energy", IEEE Power and Energy Magazine; pp.16-18, 2003.

[2] Riffat SB, Ma X. "Thermoelectrics: a review of present and potential applications." Applied Thermal Engineering. pp. 13-35, 2003.

[3] Simons RE, Ellsworth MJ, Chu RC. "An assessment of module cooling enhancement with thermoelectric coolers.” J Heat Transfer-Trans ASME. pp. 76-84, 2005.

[4] Wu KH, Hung CI. "Thickness scaling characterization of thermoelectric module for small-scale electronic cooling.” J Chin Soc Mech Eng. pp. 75-81, 2009.

[5] Cheng TC, Cheng CH, Huang ZZ, Liao GC. "Development of an energy-saving module via combination of solar cells and thermoelectric coolers for green building applications." Energy. pp. 3340, 2011.

[6] Chen WH, Liao CY, Hung CI. "A numerical study on the performance of miniature thermoelectric cooler affected by Thomson effect.” Appllied Energy. pp. 64-73, 2012.

[7] Champier D, Bedecarrats JP, Rivaletto M, Strub F. "Thermoelectric power generation from biomass cook stoves.” Energy. pp. 35-42, 2010.

[8] Champier D, Bedecarrats JP, Kousksou T, Rivaletto M, Strub F, Pignolet P. "Study of a TE (thermoelectric) generator incorporated in a multifunction wood stove." Energy. pp. 18-26, 2011.

[9] Martinez A, Astrain D, Rodriguez A. "Experimental and analytical study on thermoelectric self cooling of devices.” Energy. pp. 50-60, 2011.

[10] Chen M, Rosendahl LA, Condra T. "A three-dimensional numerical model of thermoelectric generators in fluid power systems.” Int J Heat Mass Transfe. pp. 45-55, 2011.

[11] Amatya R, Ram RJ. "Solar thermoelectric generator for micropower applications." Journal of Electronic Materials. pp. 35-40, 2010.

[12] Esarte J, Min G, Rowe DM. "Modelling heat exchangers for thermoelectric generators." J Power Sources. pp. 2-6, 2001.

[13] Yamashita O. "Effect of linear temperature dependence of thermoelectric properties on energy conversion efficiency." Energy Conversion and Management . pp. 3-9, 2008.

[14] Yilbas BS, Sahin AZ. "Thermoelectric device and optimum external load parameter and slenderness ratio". Energy. pp. 80-84, 2010.

[15] Gaowei Liang, Jiemin Zhou, Xuezhang Huang. Analytical model of parallel thermoelectric generator.”; Applied Energy 88. pp. 5193-5199, 2011

[16] Liang GW, Zhou JM, Huang XZ, et al. "Analytical model of series semiconductor thermoelectric generators.”; J Jiangsu Univ Sci Technol, pp. 4-9, 2011. 
[17] Zhang HJ, Chen H, et al. "Research on the generating performance of seriesparallel connection and reappearance of a semiconductor thermoelectric module."; Acta Energiae Solaris Sinica. pp. 4-7, 2001.

[18] Hsiao YY, Chang WC, Chen SL. "A mathematic model of thermoelectric module with applications on waste heat recovery from automobile engine.” Energy. pp. 47-54, 2010.

[19] Hsu CT, Huang GY, Chu HS, et al. "Experiments and simulations on lowtemperature waste heat harvesting system by thermoelectric power generators." Appllied Energy. pp. 1-7, 2011.

[20] Masahide M, Michio M, Masaru O. "Thermoelectric generator utilizingautomobile engine exhaust gas." Therm Sci Eng. pp. 7-8, 2001.

[21] Yodovard P. "The potential of waste heat thermoelectric power generation from diesel cycle and gas turbine cogeneration plants." Energy Source. pp. 3-4, 2001.

[22] Yu C, Chau KT. "Thermoelectric automotive waste heat energy recovery using maximum power point tracking." Energy Convers Manage. pp. 6-12, 2009.

[23] Xu LZ, Li Y, Yang Z, et al. "Experimental study of thermoelectric generation from automobile exhaust." J Tsinghua Univ (Sci Technol). pp. 7-9, 2010.

[24] Li P, Cai L, Zhai P, Tang X, Zhang Q, Niino M. "Design of concentration solarthermoelectric generator." Journal of Electronic Materials . pp. 22-30, 2010.

[25] Wei He, Yuehong Su, S.B. Riffat, JinXin Hou, Jie Ji. "Parametrical analysis of the design and performance of a solar heat pipe thermoelectric generator unit." Applied Energy 88. pp. 5083-5089, 2011.

[26] Eberhart, R. C., Kennedy, J. "A new optimizer using particle swarm theory” [A]. In: Proceedings of the Sixth International Symposium on Micromachine and Human Science [C]. Nagoya, Japan, pp. 3943, 1995.

[27] Clerc, M. (1999). "The Swarm and the queen: towards a deterministic and adaptive particle swarm optimization.” Proc. 1999 Congress on Evolutionary Computation, Washington, DC, pp 1951-1957. Piscataway, NJ: IEEE Service Center.

[28] Christophe Goupil, Wolfgang Seifert, Knud Zabrocki, Eckhard Muller and G. Jeffrey Snyder; "Thermodynamics of Thermoelectric Phenomena and Applications"; Entropy. pp. 1481-1517, 2011.

[29] Melcor Materials Electronic Products Corporation Product Specifications. NJ: Treton; 1992.

[30] Eberhart, R. C., Kennedy, J. A new optimizer using particle swarm theory [A]. In: Proceedings of the Sixth International Symposium on Micromachine and Human Science [C]. Nagoya, Japan, 1995. 